\title{
Geração de créditos de carbono na queima de metano na carbonização
}

\author{
Júlia Melo Franco Neves Costa ${ }^{1 \star}$, Fabiana Paiva de Freitas ${ }^{1}$, Wagner Davel Canal ${ }^{1}$, Mateus \\ Alves de Magalhães ${ }^{1}$, Ana Márcia Macedo Ladeira Carvalhoํㅜ, Renato Vinícius Oliveira Castro² \\ ${ }^{1}$ Universidade Federal de Viçosa (UFV), Viçosa, Minas Gerais, Brasil. \\ ${ }^{2}$ Universidade Federal de São João del-Rei (UFSJ), São João del-Rei, Minas Gerais, Brasil.
}

\begin{abstract}
RESUMO Este estudo foi realizado com o objetivo de avaliar a redução de metano $\left(\mathrm{CH}_{4}\right)$ obtida com a queima dos gases oriundos do processo de carbonização. Foram realizadas duas carbonizações, com e sem a queima dos gases, a fim de se determinar a redução da emissão do gás $\mathrm{CH}_{4}$. Para a obtenção da vazão mássica do referido gás foram combinadas as variáveis ambientais de temperatura e pressão com as variáveis do processo de carbonização, como a pressão diferencial do fluxo e vazão volumétrica dos gases. Como resultados, obteve-se uma combustão eficiente dos gases de efeito estufa, com redução equivalente de 99,8\% do gás $\mathrm{CH}_{4}$. Além disso, em um ano, observou-se uma redução de 21,45 ton. $\mathrm{CO}_{2}$ e, o que corresponde a uma receita anual em créditos de carbono de $\mathrm{R} \$ 33,50$. Pode-se concluir que a utilização de uma fornalha acoplada ao forno de carbonização possui contribuição efetiva na mitigação da emissão dos gases do efeito-estufa.
\end{abstract}

Palavras-chave: Gases de efeito-estufa; dióxido de carbono equivalente; carvão vegetal.

\section{Generation of carbon credits during methane burning through carbonization}

\begin{abstract}
This study was carried out to evaluate the reduction of methane $\left(\mathrm{CH}_{4}\right)$ obtained by the burning of gases during the carbonization process. Two carbonizations were carried out, with and without the burning of the gases, to determine the reduction of $\mathrm{CH}_{4}$ emission. To obtain the mass flow rate of the gas, environmental variables of temperature and pressure were combined with the variables of the carbonization process, such as the differential pressure of the gaseous flow and volumetric flow. As results, an efficient combustion of the greenhouse gases was obtained, with the reduction equivalent to $99.8 \%$. In addition, over a year, there was a reduction of 21.45 ton. $\mathrm{CO}_{2} \mathrm{e}$, which corresponds to an annual revenue of carbon credits of $\mathrm{R} \$$ 33.50. It can be concluded that the use of a furnace coupled to the carbonization furnace is an effective contribution to the mitigation of greenhouse gas emissions.
\end{abstract}

Keywords: Greenhouse gases; carbon dioxide equivalent; charcoal.

\section{Introdução}

Discussões com enfoque na temática ambiental, em especial relacionadas à mudança do clima, têm estado em grande evidência nos últimos anos, dado a influência direta dos setores produtivos na intensificação do processo de efeito estufa global. Em virtude das alterações climáticas ocasionadas pela emissão de gases de efeito estufa (GEE) em diversos setores de produção, preocupações relativas à preservação do meio ambiente estimularam a criação do mecanismo de desenvolvimento limpo (MDL), ferramenta de flexibilização proposta pelo Protocolo de Quioto. De acordo com o Ministério de Ciência e Tecnologia (MCTI, 2016), o MDL prevê a venda de reduções de emissões de GEE em projetos de pequena e grande escala em países em desenvolvimento que produzem energia sob baixa emissão de $\mathrm{CO}_{2}$ na atmosfera. 
Com grande potencial de geração de energia limpa, a produção de carvão vegetal no Brasil pode ser considerada sustentável, uma vez que se usa madeira proveniente de reflorestamentos para o carvoejamento. Apesar de o carvão vegetal ser originado de madeira oriunda de plantios florestais sustentáveis, constata-se que o processo de carbonização, realizado pela maioria dos pequenos produtores brasileiros, é caracterizado pela baixa eficiência energética e não recuperação dos GEE’s liberados. Nesse contexto, a utilização de mecanismos de flexibilização, como o MDL, viabilizaria a adoção de práticas operacionais mais eficientes e o emprego de melhores tecnologias na produção de carvão vegetal. Além disso, a melhora operacional na carbonização pode possibilitar o aproveitamento dos produtos derivados do processo e a redução das emissões dos gases de efeito estufa, principalmente o gás $\mathrm{CH}_{4}$. Uma das justificativas para se evitar as emissões do gás $\mathrm{CH}_{4}$ durante a produção de carvão vegetal seria que o seu potencial poluidor é, segundo Forster et al. (2007), 25 vezes maior que o gás $\mathrm{CO}_{2}$, considerado como gás de efeito estufa referência.

Diante da problemática inserida, uma das ferramentas que vêm sendo utilizadas atualmente no Brasil para se mitigar os impactos negativos da produção de carvão vegetal é a construção de fornalhas ou queimadores de gases. Segundo Cardoso (2010b), tais instrumentos podem ser utilizados para se evitar a emissão de gases poluentes para a atmosfera durante a carbonização da madeira e auxiliam na melhora da qualidade do carvão produzido. Portanto, ao se inserir a nova proposta de produção de carvão vegetal, caracterizada pela queima dos GEE's, o MCTI (2016), garante que somente a não emissão do gás $\mathrm{CH}_{4}$ no processo de carbonização implica no cumprimento dos compromissos de redução de GEE pelo Protocolo de Quioto.

Em virtude do que foi exposto sobre o potencial poluidor do gás $\mathrm{CH}_{4}$ e a redução de sua emissão durante a carbonização da madeira, percebe-se a necessidade de avaliar a eficiência da metodologia AMSIIIK (UNFCCC, 2016) e destacar a sua utilização para um importante nicho da cadeia do carvão vegetal, o dos pequenos produtores. Diante disso, o objetivo do presente trabalho foi avaliar a queima dos gases oriundos do processo de carbonização em forno de alvenaria como base à geração de créditos de carbono para projetos de pequena escala.

\section{Material e Métodos}

\section{Material biológico}

Utilizou-se madeira de Eucalyptus spp., com aproximadamente 8 anos de idade, provenientes de plantios comerciais de uma empresa florestal situada no município de Paula Cândido, Minas Gerais (MG).

\section{Ensaios de carbonização}

As carbonizações em forno de alvenaria foram realizadas de tal forma a simular um projeto de produção de carvão vegetal em pequena escala, estimando a redução de emissão dos gases de efeito estufa, em especial a do gás $\mathrm{CH}_{4}$ ) com a utilização de fornalha para combustão dos gases gerados, como ilustrado na Figura 1.

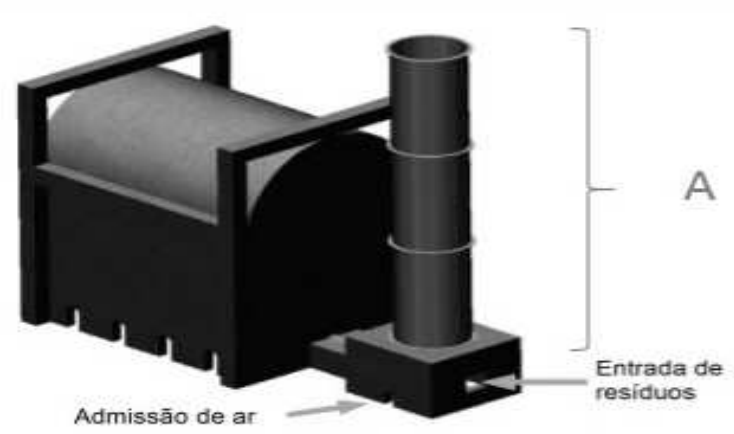

Figura 1. Vista em perspectiva do sistema forno-fornalha. Figure 1. Perspective view of furnace system.

O forno utilizado possuía quatro aberturas de cada lado em sua base, sendo duas de maior dimensão em sua parte 
frontal destinada à ignição do processo, e as demais para controle da carbonização. A fornalha utilizada para a queima dos gases possuía dimensões externas de $1 \mathrm{~m} \mathrm{x} 1 \mathrm{~m}$, e aberturas laterais junto à base de $0,12 \mathrm{~m}$ x $0,12 \mathrm{~m}$ para admissão de ar e frontal de $0,30 \mathrm{~m}$ x 0,30 m para adição de resíduos. A câmara de combustão da fornalha e a chaminé uniam-se em um único conjunto cilíndrico, formado por módulos de aço conectados por flanges, com um diâmetro interno de $60 \mathrm{~cm}$ e altura total de $460 \mathrm{~cm}$, como apontado em A na Figura 1.

A conexão entre o forno e a fornalha foi feita por um duto metálico com $0,30 \mathrm{~m}$ de diâmetro interno. $\mathrm{O}$ controle da vazão dos gases se deu por válvulas do tipo "borboleta" e "guilhotina", caracterizadas por chapas metálicas instaladas no interior do duto, com movimento horizontal e vertical, respectivos, com o intuito de restringir a vazão dos gases de acordo com seus ângulos de abertura.

O conjunto formado pela câmara de combustão e a chaminé foi revestido internamente com manta cerâmica (constituída por um complexo químico de $35 \%$ de $\mathrm{Al}_{2} \mathrm{O}_{3}, 50 \%$ de $\mathrm{SiO}_{2}, 15 \%$ de Zirconia e $0,05 \%$ de $\mathrm{Fe}_{2} \mathrm{O}_{3}, \mathrm{CaO}$ e $\mathrm{MgO}$ ) aderida por argamassa refratária, a fim de reduzir a perda de calor no interior da fornalha durante a queima dos gases, bem como retardar a depreciação dos materiais utilizados.

\section{Coleta e caracterização dos gases}

\section{Instrumentação do forno}

Foram considerados dois pontos de aquisição dos dados de concentração, temperatura e pressão dos gases, sendo um no duto de conexão do forno com a fornalha, e outro na chaminé, ilustrado na Figura 2A.

Nas Figuras 2B e 2C a instrumentação no duto de saída dos gases e na chaminé era composta por termoresistores, termopares e pontos de coleta de gases. A pressão dinâmica foi determinada por meio de um sistema de pitot, que se estimou a velocidade de escoamento do fluido e se obteve um valor de vazão, utilizando a área da secção da tubulação de escape. A análise das emissões gasosas do processo de carbonização foi realizada por fração volumétrica dos gases, medida utilizando um sistema de análise de gases (Gasboard 3100), o qual forneceu leituras da composição percentual de metano $\left(\mathrm{CH}_{4}\right)$, dióxido de carbono $\left(\mathrm{CO}_{2}\right)$, monóxido de carbono (CO), hidrogênio $\left(\mathrm{H}_{2}\right)$, oxigênio $\left(\mathrm{O}_{2}\right)$ e hidrocarbonetos de cadeia curta $\left(\mathrm{C}_{\mathrm{n}} \mathrm{H}_{\mathrm{m}}\right)$. Tem-se o suporte das tecnologias NDIR (Non - Dispersive Infrared) para se realizar as leituras de concentração dos gases $\mathrm{CO}, \mathrm{CO}_{2}$ e $\mathrm{CH}_{4}$ e TCD (Thermal Conductivity Detector) e ECD (Electron Capture Detector) para as concentrações de $\mathrm{H}_{2}$ e $\mathrm{O}_{2}$, conforme apresentado na Tabela 1.

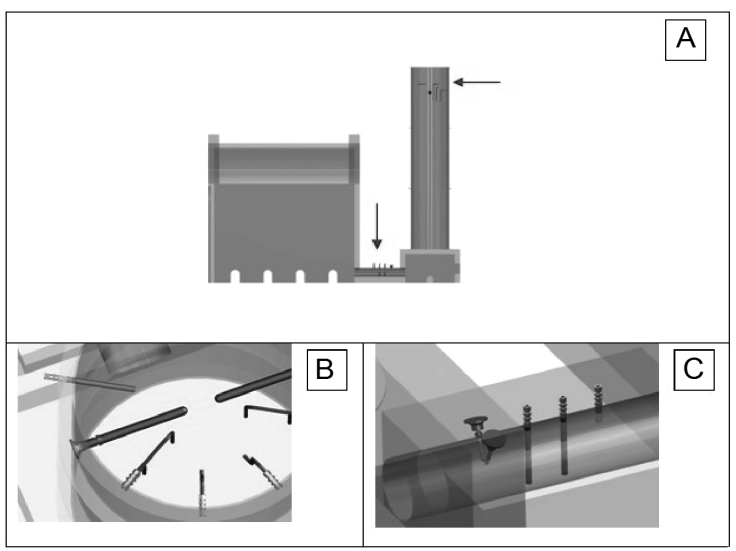

Figura 2. Modelo do forno (A) e pontos de instrumentação na saída da chaminé (B) e na saída do forno (C).

Figure 2. Model of oven (A) and instrumentation points at chimney outlet (B) and oven outlet (C).

Para a mensuração dos dados coletados (em gramas), fezse necessária coleta das condições ambientais de pressão, temperatura e umidade. Para a coleta da temperatura ambiente utilizou-se o sensor tipo CI LM35, que tem incerteza de $0,5^{\circ} \mathrm{C}$ e faixa de -55 a $150^{\circ} \mathrm{C}$. A pressão ambiente foi obtida através de um transdutor piezo-resistivo, com faixa de medição de 0-200 kPa e um erro máximo de 2,5\%. Por fim, a umidade relativa foi determinada pelo sensor capacitivo 
HIH4004, que possui erro máximo de 2\%. A medição foi realizada de forma contínua, gerando uma amostragem de alta frequência para o conteúdo gasoso, temperatura e vazão, de forma a caracterizar inteiramente o processo.

Tabela 1. Especificações das medições gasosas.

Table 1. Gas measurement specifications.

\begin{tabular}{ccccc}
\hline Gás & Método & Faixa & Resolução & Incerteza \\
\hline $\mathrm{CO}_{2}$ & NDIR & $0-25 \%$ & $0,01 \%$ & $\leq 2 \%$ \\
\hdashline $\mathrm{CO}$ & NDIR & $0-75 \%$ & $0,01 \%$ & $\leq 2 \%$ \\
\hdashline $\mathrm{CH}_{4}$ & NDIR & $0-25 \%$ & $0,01 \%$ & $\leq 2 \%$ \\
\hdashline $\mathrm{H}_{2}$ & $\mathrm{TCD}$ & $0-40 \%$ & $0,01 \%$ & $\leq 3 \%$ \\
\hdashline $\mathrm{O}_{2}$ & $\mathrm{ECD}$ & $0-25 \%$ & $0,01 \%$ & $\leq 3 \%$ \\
\hline
\end{tabular}

\section{Método de cálculo}

A sequência para obtenção da vazão mássica de cada gás de interesse pode ser observada na Figura 2.

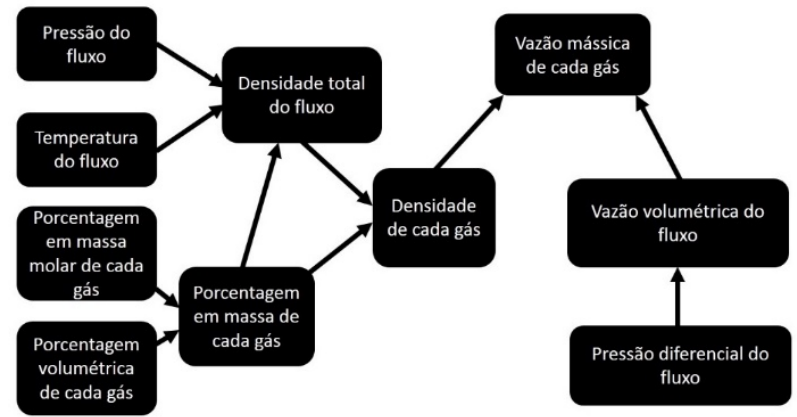

Figura 3. Fluxograma da rotina de cálculo dos parâmetros de interesse.

Figure 3. Flowchart of the routine of calculation of the parameters of interest.

Por meio da pressão diferencial do fluxo, foi possível encontrar a velocidade de escoamento do mesmo. Tendo-se as características dimensionais da tubulação, determinou-se a vazão volumétrica do fluxo de gás. A partir da porcentagem em massa molar de cada gás medido, em conjunto com o percentual em volume dos mesmos, calculou-se a porcentagem em massa de cada gás presente no fluxo. Ao se combinar os dados citados anteriormente com as condições de temperatura e pressão do fluxo, obtiveram-se as densidades total e parciais do fluxo, que juntamente com as vazões volumétricas, resultaram nas vazões mássicas dos gases.

\section{Simulação da redução de gases de efeito estufa}

Com base na metodologia AMSIIIK (UNFCCC, 2016), considerou-se como cenário linha de base, a carbonização conduzida sem promover a queima dos gases na fornalha, tal como é feito nos métodos tradicionais frequentemente utilizados. Já no cenário alvo, considerou-se a carbonização em que se promoveu a combustão dos gases no interior da fornalha.

\section{Redução das emissões}

A estimativa da redução das emissões decorrente da implementação do projeto, foi calculada pela diferença entre as principais emissões de gases de efeito estufa observadas durante a produção de carvão vegetal para a linha de base e as principais emissões de gases de efeito estufa observadas para o projeto, conforme Equação 1.

$$
R E=E B-(E P+F u g a)
$$

Onde: $\mathrm{RE}=$ Redução das emissões em um ano (ton. $\mathrm{CO}_{2} \mathrm{e}$ ); $\mathrm{EB}$ $=$ Emissões da linha de base durante um ano (ton. $\mathrm{CO}_{2} \mathrm{e}$ ); $\mathrm{EP}=$ Emissões diretas das atividades do projeto durante um ano (ton. $\mathrm{CO}_{2} \mathrm{e}$ ); Fuga = Emissões por substituição de tecnologia ultrapassada (ton. $\mathrm{CO}_{2} \mathrm{e}$ ).

\section{Emissões da linha de base (EB)}

A forma de cálculo para as emissões gasosas da linha de base anterior aos processos de melhoria do forno está exposta na Equação 2.

$$
E B=Q m p * M * G W P C H
$$

Onde: $\mathrm{EB}_{\mathrm{y}}=$ Emissões da linha de base em um ano (ton. $\left.\mathrm{CO}_{2} \mathrm{e}\right) ; \mathrm{Q}_{\mathrm{mp}, \mathrm{y}}=$ Quantidade de matéria-prima em base seca (ton.) utilizada nas novas instalações durante um ano; $\mathrm{M}=$ Fator de emissão do $\mathrm{CH}_{4}$ para fabricação de carvão no 
método convencional considerado (ton. $\mathrm{CH}_{4} /$ ton. de matéria-prima); $\mathrm{GWPCH}_{4}=$ Potencial de Aquecimento Global do gás $\mathrm{CH}_{4}$.

\section{Emissões do projeto (EP)}

A forma de cálculo para as emissões gasosas da atividade do projeto está exposta na Equação 3.

$$
\begin{aligned}
& \text { EPy }=\text { EPtransp. } 1+\text { EPtransp. } 2+ \\
& \text { EPeletr. }+ \text { EPfugitivo }+ \text { EPapoio }
\end{aligned}
$$

Onde: $\mathrm{EPy}=$ Emissões diretas das atividades do projeto em um ano (ton. $\mathrm{CO}_{2} \mathrm{e}$ ); $\mathrm{EP}_{\text {transpl }}=$ Emissões de transporte nos pontos de coleta de matéria-prima em um ano (ton. $\mathrm{CO}_{2} \mathrm{e}$ ); $\mathrm{EP}_{\text {transp2 }}=$ Emissões de transporte nos pontos de consumo em um ano (ton. $\mathrm{CO}_{2} \mathrm{e}$ ); $\mathrm{EP}_{\text {eletr. }}=$ Emissões de eletricidade ou de consumo de diesel em um ano (ton. $\mathrm{CO}_{2} \mathrm{e}$ ); $\mathrm{EP}_{\text {fugitivo }}=\mathrm{As}$ emissões fugitivas de captura e queima ineficiente no projeto de fabricação do carvão durante um ano (ton. $\mathrm{CO}_{2} \mathrm{e}$ ); $\mathrm{EP}_{\text {apoio }}=$ As emissões de combustíveis fósseis, para manter a incineração do gás de carbonização, durante um ano (ton. $\left.\mathrm{CO}_{2} \mathrm{e}\right)$.

\section{Créditos de carbono}

Com base no resultado da redução das emissões alcançada durante o período de um ano, em toneladas de $\mathrm{CO}_{2} \mathrm{e}$, tornouse possível estimar a receita adquirida com a comercialização dos Certificado de Emissões Reduzidas (CER's), de acordo com a cotação da tonelada de $\mathrm{CO}_{2} \mathrm{e}$. Assim, com o fator de redução de emissão obtido neste estudo $\left(\mathrm{kg} \mathrm{CO}_{2} \mathrm{e} /\right.$ ton. madeira) foi possível estimar a redução dos gases de efeito estufa na produção de carvão vegetal durante um ano.

\section{Resultados e Discussão}

\section{Eficiência de queima dos GEE}

Com a presente metodologia aplicada ao estudo, encontrou-se que a emissão do gás $\mathrm{CH}_{4}$ na carbonização sem a queima dos gases foi de 7,58 $\mathrm{kg}$ para cada tonelada de madeira seca enfornada. Ao se extrapolar a emissão do gás $\mathrm{CH}_{4}$ para a carbonização de 2,36 toneladas de madeira seca enfornada no presente estudo, encontrou-se um fator de emissão de 17,88 kg. Já considerando a carbonização com a queima dos gases oriundos do processo, na qual se utilizou 2,36 toneladas de madeira seca, foram emitidos $0,0236 \mathrm{~kg}$. Portanto, comparando os valores de carbonização sem queima e com queima dos gases, percebe-se uma combustão eficiente dos mesmos, pois em relação ao $\mathrm{CH}_{4}$ houve uma redução equivalente a 99,8\% de emissão em massa.

Tais dados estão em consonância com os encontrados por Cardoso et al., (2010a), que relataram uma redução de 96\% do $\mathrm{CH}_{4}$ após a queima dos gases. Já em estudos promovidos por Halouani; Farhat (2003), as emissões dos gases combustíveis oriundos da carbonização da madeira foram completamente eliminadas, numa planta de quatro fornos metálicos ligados a uma unidade de queima.

\section{Emissões da linha de base (EB)}

As emissões da linha de base durante um ano para o gás $\mathrm{CH}_{4}$, principal gás de efeito estufa, está prevista na Equação 2 descrita no item 2.4.2. Logo, a massa de madeira utilizada para a carbonização de 2,36 ton. em um ciclo de 4 fornadas mensais durante 12 meses é de 113,2 toneladas. O fator de emissão de $\mathrm{CH}_{4}$ para a produção de carvão pelo método convencional, descrito no item 3.1, foi de 0,00758 ton. $\mathrm{CH}_{4} /$ ton. mad. E, segundo Forster et al. (2007), o potencial de aquecimento global (GWP) considerado para o gás $\mathrm{CH}_{4}$ é de 25. Dessa forma, as emissões da linha base durante um ano foram estimadas em 21,45 ton. $\mathrm{CO}_{2} \mathrm{e}$.

Portanto, de acordo com o potencial de aquecimento global descrito para o gás $\mathrm{CH}_{4}$, pode-se verificar que a sua emissão para a atmosfera através da carbonização da madeira impacta significativamente para a depreciação do meio ambiente (FORSTER et al., 2007). Em virtude disso, a utilização de protótipos de fornalhas, como o descrito neste estudo, é vista como alternativa para a produção de carvão 
vegetal sem grandes emissões de gases de efeito estufa para o ambiente, dado a sua elevada eficiência na queima. Além disso, Miranda et al. (2013) mencionam que o uso de tecnologias de queima de gases na carbonização da madeira pode promover a geração de energia térmica para a secagem de grãos e até mesmo a cogeração de energia elétrica.

\section{Emissões do projeto (EP)}

Para a mensuração das emissões dadas à implementação do projeto, deve-se considerar que as variáveis expostas na Equação 3 possuem determinadas particularidades neste estudo. As emissões relativas ao transporte da madeira e do carvão vegetal ( $\mathrm{EP}_{\text {transp.1 }}$ e $\left.\mathrm{EP}_{\text {transp. } 2}\right)$ foram desprezadas, uma vez que não há distância incremental para a implementação do projeto em relação à linha de base. Também as emissões de eletricidade ( $\left(\mathrm{PP}_{\text {eletr. }}\right)$ foram consideradas nulas, pois o projeto proposto não prevê consumo extra de energia, seja com novas instalações ou com periféricos para o forno. Em relação às emissões de combustíveis gastos para manter a incineração dos gases na fornalha $\left(\mathrm{EP}_{\text {apoio }}\right)$, estes se referem a combustíveis fósseis e não se aplicam ao estudo em questão, visto que se utilizou apenas combustíveis de origem renovável. É válido ressaltar, no entanto, que foram gastos aproximadamente $150 \mathrm{Kg}$ de biomassa, principalmente atiços, para manutenção da chama na fornalha, notadamente nas primeiras horas de carbonização, correspondente à fase de secagem da madeira.

Portanto, as emissões do projeto no ano correspondem apenas às emissões de captura e queima ineficientes do $\mathrm{CH}_{4}$ $\left(\mathrm{EP}_{\text {fugitivo }}\right)$, cuja equação resultante pode ser desmembrada a seguir.

$$
E P=1-(\text { CFEproj. }) * E M p r o j . * G W P C H 4
$$

Onde: $\mathrm{CFE}_{\text {proj. }}=$ Eficiência do dispositivo de captura e queima do $\mathrm{CH}_{4} ; \mathrm{EM}_{\text {proj. }}=$ Emissões diretas do projeto em um ano; $\mathrm{GWP}_{\mathrm{CH} 4}=$ Potencial de Aquecimento Global do $\mathrm{CH}_{4}$.
$\mathrm{Na}$ Equação 4 está presente o valor de $\mathrm{CFE}_{\text {proj. }}$, que corresponde à eficiência do equipamento de captura e queima do $\mathrm{CH}_{4}$, considerado 0,9 , valor padrão estabelecido pela metodologia AMSIIIK. Consideram-se também as emissões diretas do projeto $\left(\mathrm{EM}_{\text {projeto }}\right)$, correspondentes ao produto dos 0,0236 $\mathrm{kg}$ de $\mathrm{CH}_{4}$ por quatro carbonizações ao mês, totalizando 48 ao ano, e o Potencial de Aquecimento Global do $\mathrm{CH}_{4}$, que corresponde a 25. Portanto, as emissões do projeto somaram 2,832 $\mathrm{kg}$ de $\mathrm{CO}_{2} \mathrm{e}$ ou 0,002832 ton. de $\mathrm{CO}_{2} \mathrm{e}$, valor ínfimo que reflete a eliminação de quase a totalidade do $\mathrm{CH}_{4}$ com a queima dos gases da carbonização.

Destaca-se que o potencial de emissão de gases de efeito estufa pode ser evitado não somente com a implementação de novas tecnologias para produção de carvão vegetal, como também, segundo destacado por Canal et al. (2016), deve-se atentar ao manejo correto do teor de umidade da biomassa previamente à sua carbonização. Ainda de acordo com Njenga et al. (2014), faz-se necessário observar que se a madeira usada para tal produção vier de florestas plantadas, o $\mathrm{CO}_{2}$ total emitido durante a carbonização será totalmente absorvido pelo crescimento de novas árvores, tornando o processo sustentável.

\section{Redução das emissões e obtenção de créditos de carbono}

De posse dos valores das emissões da linha de base (EB) e das emissões do projeto (EP), procedeu-se ao cálculo para estimar a redução de emissões alcançadas em um ano, conforme demonstrado na Tabela 1.

Tabela 1. Redução das emissões dos gases no período de um ano.

Table 1. Reduction of emissions for one year.

\begin{tabular}{|c|c|c|c|c|c|}
\hline $\mathrm{RE}$ & & $\mathrm{EB}$ & & EP & Fuga \\
\hline$\left(\mathrm{tCO}_{2} \mathrm{e}\right)$ & $=$ & $\left(\mathrm{tCO}_{2} \mathrm{e}\right)$ & - & $\left(\mathrm{tCO}_{2} \mathrm{e}\right)$ & $\left(\mathrm{tCO}_{2} \mathrm{e}\right)$ \\
\hline 21,448 & $=$ & 21,45 & - & 0,00282 & 0 \\
\hline
\end{tabular}

Onde: $\mathrm{RE}=$ Redução de emissões em um ano; $\mathrm{EB}=$ Emissões da linha de base em um ano; EP = Emissões do projeto atividade em um ano. 
Observa-se que a redução das emissões durante um ano foi de $21,448 \mathrm{tCO}_{2} \mathrm{e}$, que corresponde praticamente ao valor das emissões da linha base, já que as emissões do projeto foram praticamente nulas. As 21,448 $\mathrm{tCO}_{2} \mathrm{e}$ reduzidas durante um ano com a implementação do projeto equivalem ao mesmo valor em CERs. Considerando um preço de $€ 0,40$ por tonelada de $\mathrm{tCO}_{2} \mathrm{e}$ (ICE, 2016), a receita em créditos carbono estimada com a utilização de fornalha para a queima de gases é de $€ 8,58$, equivalente a aproximadamente $R \$ 33,50$. Num horizonte de dez anos, período para o qual um projeto for aprovado para receber créditos de carbono, a receita total seria de aproximadamente $\mathrm{R} \$ 335,00$ por forno. Dessa forma, pode-se considerar que a redução das emissões de gases de efeito estufa seja expressiva em termos de sustentabilidade. Já a receita com a comercialização dos CERs apresenta-se como um valor irrisório, refletindo a baixa cotação atual dos créditos de carbono, principalmente quando se considera os custos referentes à elaboração do projeto.

Em relação aos custos de confecção do projeto, são contabilizadas as despesas relativas à elaboração do Documento de Concepção do Projeto (DCP), validação, aprovação, registro, monitoramento, verificação e registro, cuja soma corresponde à quantia de US\$50.000,00, considerando os custos mínimos de cada etapa definidos pelo Centro de Gestão e Estudos Estratégicos (2008). Para arcar com esse valor, seria necessário contabilizar a receita referente à redução conjunta de aproximadamente 2.985 fornos do mesmo porte deste estudo, o que se torna um grande entrave à inserção do pequeno produtor de carvão vegetal nos moldes do MDL, visto que, em média, cada proprietário possui aproximadamente 12 fornos por unidade produtora de carvão (UPC). Apesar da atual desvalorização da cotação do $\mathrm{CO}_{2} \mathrm{e}$, deve-se dar grande importância aos projetos de produção de carvão vegetal de pequena escala, com a valorização do preço pago pelos CERs e criação de uniões entre os produtores para elaboração de um projeto em comum, em forma de associações ou cooperativas, por exemplo. Segundo Torres (2011), a união entre os pequenos produtores de carvão vegetal em forma de cooperativas é uma prática aceita pela UNFCCC e já vem acontecendo em países como a Índia, Bolívia e Paraguai e recebem auxílio internacional para que as pequenas propriedades destes países pleiteiem créditos de carbono.

Um dos incentivos para a criação de uniões de pequenos produtores em sindicatos é a presença de um grande mercado consumidor de carvão vegetal que se encontra no Brasil. Somente no estado de Minas Gerais, o consumo de carvão vegetal no ano de 2011 pelo segmento de produção independente de ferro-gusa foi de 8.994.720 mdc (AMS, 2012). Portanto, ao se considerar o elevado consumo de carvão vegetal e a formação de sindicatos de produtores, pode-se garantir sustentabilidade à siderurgia nacional, dado a mitigação dos danos ambientais provocados pela não emissão de $\mathrm{CH}_{4}$ na atmosfera e retornos financeiros complementares à produção de carvão.

Outro fator que deve ser levado em consideração durante a produção de carvão vegetal seria a mudança do perfil potencialmente poluidor dessa prática para a inserção do Brasil numa economia de baixo carbono. Dessa maneira, o aspecto da produção de carvão vegetal não seria visto exclusivamente por meio da geração de créditos de carbono, mas também pela gestão dos gases de efeito estufa dentro das organizações, formulação de leis estaduais e federais específicas, obtenção de linhas de financiamento especiais, dentre outros (ICF, 2010). Portanto, deve-se estimular o desenvolvimento de projetos de baixo carbono, com foco ou não no MDL. Uma opção para o incentivo de redução das emissões de GEE durante a carbonização seria utilizar os princípios da neutralização do carbono. Assim, promovendose a queima dos gases do processo de carbonização, ela estaria 
neutralizando as emissões de outras atividades, como por exemplo, a utilização de combustíveis fósseis em motores. Dessa forma, no carvão produzido pode-se utilizar o rótulo de "carbono zero", promovendo um "marketing ambiental", que poderia ser responsável pela conquista de mercado, principalmente internacional.

\section{Conclusões}

A eficiência de queima do gás $\mathrm{CH}_{4}$ pela fornalha acoplada ao forno foi de $99,8 \%$ em massa. Além da queima dos gases da carbonização auxiliar na melhora das condições de trabalho para as pessoas que operam os fornos de carbonização, a energia térmica liberada nessa ocasião pode ser aproveitada para a secagem da lenha ou ainda na cogeração de energia elétrica.

Pode-se propor a criação de uniões (ou cooperativas) entre produtores de carvão vegetal para a elaboração de um projeto de redução de gases de efeito estufa em comum, com o intuito de garantir maiores lucros com a mitigação das emissões de gases de efeito estufa.

\section{Agradecimentos}

Os autores agradecem à Fundação de Amparo à Pesquisa do Estado de Minas Gerais - FAPEMIG, ao Conselho Nacional de Pesquisa e Desenvolvimento - CNPq e à Secretaria de Estado de Ciência, Tecnologia e Ensino Superior - SECTES pelo apoio financeiro; e à Embrapa Florestas pelo apoio dado para realização desse trabalho.

\section{Referências}

AMS - ASSOCIAÇÃO MINEIRA DE SILVICULTURA. Anuário estatístico 2011. Belo Horizonte, 2012.

CANAL, W. D.; CARVALHO, A. M. M. L.; CARNEIRO, A. C. O.; VITAL, B. R.; PEREIRA, B. L. C.; DONATO, D. B. Efeito do teor de umidade da madeira na emissão de gases de efeito estufa no processo de carbonização. Scientia Forestalis, Piracicaba, v. 44, n. 112, p. 831-840, 2016.

CARDOSO, M. T.; DAMASIO, R. A. P.; CARNEIRO, A. C. O.; JACOVINE, L. A. G.; VITAL, B. R.; BARCELOS, D. C. Construção de um sistema de queima de gases da carbonização para redução da emissão de poluentes. Cerne, Lavras, v. 16, Suplemento, p. 115-124, 2010a.

CARDOSO, M. T. Desempenho de um sistema fornofornalha para combustão de gases na carbonização da madeira. 2010. 77p. Dissertação (Mestrado em Ciência Florestal) Universidade Federal de Viçosa, Viçosa - MG. $2010 \mathrm{~b}$.

FORSTER, P. V.; RAMASWAMY, P.; ARTAXO, T.; BERNTSEN, R.; BETTS, D.W.; FAHEY, J.; HAYWOOD, J.; LEAN, D.C.; LOWE, G.; MYHRE, J.; NGANGA, R.; PRINN, G.; RAGA, M.; SCHULZ and R. VAN DORLAND. Changes in Atmospheric Constituents and in Radiative Forcing. In: Climate Change 2007: The Physical Science Basis. Contribution of Working Group I to the Fourth Assessment Report of the Intergovernmental Panel on Climate Change. Cambridge University Press, Cambridge, United Kingdom and New York, NY, USA, 2007, v. 1, p. 129234, 2007.

HALOUANI, K.; FARHAT, H. Depollution of atmospheric emissions of wood pyrolysis furnaces. Renewable Energy, v.28, n.1, p. 129-138, 2003.

ICE - Emissions CER Index. The ICE CER Futures Contract CER Index. 2016. Disponível em: $<$ http://cdm.unfccc.int/methodologies/index.html>. Acesso em: 14 jun. 2016.

ICF- Instituto Carbono Brasil. Levantamento de Oportunidades Concretas de Projetos de Baixo Carbono no Brasil. 2010.

MCTI - Ministério da ciência, tecnologia e inovação. Status dos projetos do Mecanismo de Desenvolvimento Limpo (MDL) no Brasil, 2016.

MIRANDA, R. C. de; BAILIS, R.; VIlELA, A. O. Cogenerating electricity from charcoaling: A promising new advanced technology. Energy for Sustainable Development. Amsterdã, v. 17, p. 171-176, 2013.

NJENGA, M.; KARANJA, N.; KARLSSON, H.; JAMNADASS, R.; LIYAMA, M.; KITHINJI, J. Additional cooking fuel supply and reduced global warming potential 
from recycling charcoal dust into charcoal briquette in Kenya. Journal of Cleaner Production. Amsterdã, v. 81, p. 81-88, 2014.

TORRES, C. M. M. E. Análises técnica e econômica da geração de créditos de carbono em projetos florestais na região de Viçosa, MG. Dissertação de mestrado, Universidade Federal de Viçosa, 2011. 127 p.

UNFCCC - UNITED NATIONS FRAMEWORK CONVENTION ON CLIMATE CHANGE. Avoidance of methane release from charcoal production. 30 p. 2016. 